\title{
Laser-induced thermal acoustics (LITA) signals from finite beams
}

\author{
E. B. Cummings, I. A. Leyva, and H. G. Hornung
}

\begin{abstract}
Laser-induced thermal acoustics (LITA) is a four-wave mixing technique that may be employed to measure sound speeds, transport properties, velocities, and susceptibilities of fluids. It is particularly effective in high-pressure gases ( $>1$ bar). An analytical expression for LITA signals is derived by the use of linearized equations of hydrodynamics and light scattering. This analysis, which includes full finite-beam-size effects and the optoacoustic effects of thermalization and electrostriction, predicts the amplitude and the time history of narrow-band time-resolved LITA and broadband spectrally resolved (mulitplex) LITA signals. The time behavior of the detected LITA signal depends significantly on the detection solid angle, with implications for the measurement of diffusivities by the use of LITA and the proper physical picture of LITA scattering. This and other elements of the physics of LITA that emerge from the analysis are discussed. Theoretical signals are compared with experimental LITA data.

Key words: LITA, finite-beam-size effects, thermal grating, el ectrostrictive grating, LITA vel ocimetry, multiplex LITA, data fits, dynamic light scattering.
\end{abstract}

\section{Introduction}

A four-wave-mixing technique called laser-induced thermal acoustics (LITA) has recently been used for single-shot gas property measurements ${ }^{1}$ and is the object of study at several laboratories.,3 In LITA, two beams from a narrow-band short-pulse driver laser cross, forming an electric-field grating that creates acoustic gratings through optoacoustic effects. These acoustic gratings propagate as sound waves. Through the acousto-optic effect, the sound waves scatter light from a third source laser beam into a fourth coherent signal beam, enabling spatially resolved remote measurement of gas properties. Reference 4 contains a good description of laser-induced acoustics in solids, liquids, and gases in the broader context of laser-induced grating spectroscopy.

One LITA configuration is broadband or multiplex LITA, in which the signal scattered from a broadband source beam is spectrally resolved. If the driverlaser frequency matches a transition of the medium, the signal increases because of enhanced optoacoustic effects. The LITA reflectivity when the driver laser

The authors are with the Graduate Aeronautical Laboratories, California Institute of Technol ogy, Pasadena, California 91125.

Received 19 August 1994; revised manuscript received 5 December 1994.

0003-6935/95/183290-13\$06.00/0.

(1) 1995 Optical Society of America. is resonant with a relatively strong absorption of the gas can be typically ${ }^{1} 10^{-4}$. If, in addition, the sourcelaser frequency matches a transition of the gas (not necessarily the same transition or the same species), the LITA signal increases because of enhanced acoustooptic effects. Multiplex LITA can be performed with a narrow-band driver laser resonant with a strong absorption to boost signal reflectivity and a broadband source laser tuned near spectral bands of interest. These bands need to be strong enough only to modulate the nonresonant background susceptibility. Signal detection through a spectrometer provides susceptibility spectra. The ratio of the susceptibility modulation to the nonresonant background provides absolute species concentration information, by analogy with multiplex coherent anti-Stokes Raman spectroscopy 5 (CARS). The multiplex spectra have the same shape as Rayleigh-Brillouin spectra of linear scattering.

LITA can provide fluid-velocity information. The LITA signal scattered off a convecting LITA grating bears a Doppler shift that is proportional to the local fluid velocity. Heterodyne detection of the signal recovers this Doppler shift, all owing vel ocity measurements in a manner similar to laser Doppler velocimetry. LITA velocimetry features a strong coherent signal, freedom from particle-seeding concerns, and natural application to pulsed flows.

LITA can provide gas property information, includ- 
ing temperature, transport properties, velocity, and absolute species concentration, without exhaustive study of the target species. A 30-mJ pulsed driver laser and a 1-W cw source laser have provided usable LITA signals over a range of absolute pressures of 0.4 $\mathrm{kPa}$ to $14 \mathrm{MPa}$ in $\mathrm{NO}_{2}$ and air. These signals are relatively simpleto predict and interpret. ${ }^{6}$ An expression based on the equations of linear hydrodynamics and light scattering has accurately predicted experimental results in both intensity and time history. ${ }^{1}$ This simple analysis has been extended to include finite-source-beam effects, which were important in single-shot narrow-band, time-resolved LITA experiments conducted with focused beams. The extended analysis has also uncovered interesting finite-beamsize effects and has clarified the physical picture of LITA scattering and detection.

We outline the derivation of this analytic expression for the amplitude and the time history of LITA signals. In the idealized analysis, narrow-bandwidth Gaussian driver beams generate acoustic gratings by electrostriction and thermalization, two optoacoustic effects. A Gaussian source beam scatters off these acoustic gratings into the coherent LITA signal beam. This expression is a limiting case of a more general expression for the LITA signal that is being derived, which includes the effects of laser noise, arbitrary beam profiles, fluid motion, and general optoacoustics. The analytical expression presented here illustrates effects that the general analysis obscures, while still providing good fits to experimental LITA data, several samples of which are discussed below. A discussion of the physics of LITA that emerges from the derivation, including optoacoustic grating formation, hydrodynamic evolution, signal detection effects, and an interpretation of LITA signals, accompanies the derivation.

Theanalysis presented here consists of four sections: solution of the equations governing hydrodynamic evolution in gases, modeling of optoacoustic forcing, modeling of acousto-optic scattering, and integration of the scattered field to obtain the LITA signal. The formation and the evolution of laser-induced acoustic structures are derived by the use of linear hydrodynamics with optoacoustic forcing. Simple models of thermalization and electrostriction are used. The linearized far-field equation of light scattering provides the LITA electric field scattered off acoustic gratings. Finally, the scattered field intensity integrated over a detector area yields the LITA signal intensity.

\section{Solution of Linear Equations of Grating Evolution}

LITA relies on lasers to generate nonuniformities in a fluid. The generation and the evolution of these nonuniformities modulate the LITA signal. The equations governing this evol ution fall into two categories: the hydrodynamic conservation equations that describe the bulk behavior of fluids, including acoustics and heat transfer, and diffusion equations that describe currents internal to a bulk fluid caused by species concentration gradients. Together with an equation of the state of the fluid, both sets of equations closea system for describing fluid evolution from a general laser-induced state of perturbation. The analysis presented here assumes that no species concentration perturbations are formed, which applies when the driver laser pulse is short compared with diffusive time scales and photolysis does not occur in the fluid. In this case, the governing equations degenerate to the conservation equations of hydrodynamics. The field equations in differential form for the conservation of mass (continuity), momentum, and energy in terms of perturbations in density $(\rho)$, temperature $(\mathbf{T})$, and divergence of the velocity $(\nabla \cdot \mathbf{u}) \operatorname{are}^{6,7}$

$$
\begin{gathered}
\frac{\partial \rho_{1}}{\partial \mathrm{t}}+\rho \psi_{1}=0, \\
\frac{\partial \psi_{1}}{\partial \mathrm{t}}+\frac{\mathrm{C}_{\mathrm{s}}^{2} \nabla^{2} \rho_{1}}{\gamma \rho}+\frac{\alpha \mathrm{C}_{\mathrm{s}}^{2}}{\gamma} \nabla^{2} \mathrm{~T}_{1}-\mathrm{D}_{\mathrm{V}} \nabla^{2} \psi_{1}=\dot{\psi}(\mathbf{r}, \mathrm{t} ; \mathbf{u}), \\
\frac{\partial \mathrm{T}_{1}}{\partial \mathrm{t}}-\frac{\gamma-1}{\alpha \rho} \frac{\partial \rho_{1}}{\partial \mathrm{t}}-\gamma \mathrm{D}_{\mathrm{T}} \nabla^{2} \mathrm{~T}_{1}=\dot{\mathrm{T}}(\mathbf{r}, \mathrm{t} ; \mathbf{u}),
\end{gathered}
$$

where perturbation terms are indicated by the subscript $1, \psi_{1} \equiv \nabla \cdot \mathbf{u}_{1}$, and $\nabla^{2} \equiv \partial^{2} / \partial x^{2}+\partial^{2} / \partial y^{2}+\partial^{2} / \partial z^{2}$. The parameter $\gamma$ is the ratio of specific heats of the medium $\left(c_{p} / c_{v}\right), \alpha$ is the thermal expansion coefficient $\left[-(1 / \rho)(\partial \rho / \partial T)_{p}\right], c_{s}$ is the isentropic sound speed, $D_{V}$ is the longitudinal kinematic viscosity $\left\{1 / \rho\left[\eta_{v}+(4 / 3) \eta_{s}\right]\right\}$, and $D_{T}$ is the thermal diffusivity. The forcing terms, $\dot{\psi}$ and $\mathrm{T}$, describe the optoacoustic effects. The boundary and initial conditions are

$$
\begin{aligned}
& \rho_{1}(\mathbf{r}, \mathrm{t}=0)=0, \quad \psi_{1}(\mathbf{r}, \mathrm{t}=0)=0, \mathrm{~T}_{1}(\mathbf{r}, \mathrm{t}=0)=0, \\
& \rho_{1}(\mathbf{r}, \mathrm{t}) \rightarrow 0, \quad \psi_{1}(\mathbf{r}, \mathrm{t}) \rightarrow 0, \quad \mathrm{~T}_{1}(\mathbf{r}, \mathrm{t}) \rightarrow 0, \\
& \text { as }|\mathbf{r}| \rightarrow \infty . \quad(1 \mathrm{~d})
\end{aligned}
$$

These equations apply strictly to fluids with no mean velocity. However, they are good approximations in a moving inertial frame in which the local apparent mean fluid velocity vanishes, provided that the local shear and the rotation rates are negligible. Optoacoustic forcing that rests in the laser frame moves relative to the fluid frame. Thus the forcing terms account for the local velocity. The simplified analysis presented here ignores the effects of fluid motion. In the complete analysis the effects of fluid motion are basically threefold. Cross-grating fluid motion that is comparable with the grating wavelength during the driver-laser pulse reduces the modulation depth of the grating and hence reduces the LITA reflectivity. Cross-grating fluid motion that is comparable with the source-beam diameter over the lifetime of the acoustic grating changes the 
time history of the LITA signal by changing the source-beam intensity on the grating. Finally, the driver, source, and signal beams have Doppler shifts and are nondegenerate in the fluid frame. The scattered field has a Doppler shift in the laser frame proportional to the laser-frame velocity of the grating. Optical heterodyne techniques recover this Doppler shift, permitting measurement of the cross-grating component of the gas vel ocity.

Equations (1a)-(1d) may be solved by the application of Fourier and Laplace transforms. 6,7 The acoustooptic effect responsible for the LITA signal in gases arises predominantly from density perturbations, although thermal and velocity perturbations may also contribute tothesignal. Thesolutions for the perturbation fields are

$$
\begin{aligned}
\frac{\rho_{1}(\mathbf{q}, s)}{\rho}= & M(q, s)^{-1} \\
\quad & \times\left[-c_{s} q\left(s+\gamma D_{T} q^{2}\right) \frac{\dot{\psi}}{c_{s} q}-\left(\frac{c_{s}^{2} q^{2}}{\gamma}\right) \frac{\dot{T}}{\bar{T}}\right], \\
\frac{\psi_{1}(\mathbf{q}, s)}{c_{s} q}= & M(q, s)^{-1}\left[s\left(s+\gamma D_{T} q^{2}\right) \frac{\dot{\psi}}{c_{s} q}+\left(\frac{s c_{s} q}{\gamma}\right) \frac{\dot{T}}{\bar{T}}\right], \\
\frac{T_{1}(\mathbf{q}, s)}{T}= & M(q, s)^{-1} \\
& \times\left(-(\gamma-1) s \dot{s}+\left[s\left(s+q^{2} D_{v}\right)+\frac{c_{s}^{2} q^{2}}{\gamma}\right] \frac{\dot{T}}{T}\right),
\end{aligned}
$$

where perfect gas behavior has been assumed. The quantity $M(q, s)$ is the characteristic equation of the system of al gebraic equations,

$$
\begin{aligned}
M(q, s)= & s^{3}+\left(D_{V} q^{2}+\gamma D_{T} q^{2}\right) s^{2} \\
& +\left(c_{S}^{2} q^{2}+\gamma D_{T} q^{2} D_{V} q^{2}\right) s+c_{S}^{2} q^{2} D_{T} q^{2} .
\end{aligned}
$$

Factoring $\mathrm{M}(\mathrm{q}, \mathrm{s})$ facilitates Laplace inversion. To first order in the grating Knudsen number (ratio of the mean-free path to the grating wavelength), the roots of $M(q, s)$ are approximately ${ }^{7}$

$$
\begin{aligned}
& s_{1}=-D_{T} q^{2}, \quad s_{2}=-\Gamma q^{2}+i c_{s} q, \\
& s_{3}=-\Gamma q^{2}-i c_{s} q,
\end{aligned}
$$

where $\Gamma$ is the classical acoustic damping coefficient, $\left\{1 / 2\left[(\gamma-1) D_{T}+D_{V}\right]\right\}$. The inclusion of higher-order terms is unnecessary because this first-order analysis breaks down at about the same point as the hydrodynamic equations, which assume that the gas is nearly continuous. Thus the solution is valid for acoustic disturbances with characteristic dimensions much larger than the mean-free path of the fluid; otherwise, a molecular gas-dynamics model may be needed.
For simplicity, consider only the evolution of density perturbations, $\rho_{1} / \rho$. A partial fraction expansion of the solution in the approximate roots of $M(q, s)$ elucidates the fluid behavior:

$$
\begin{aligned}
\frac{\rho_{1}}{\rho}= & {\left[\frac{\dot{T}}{\gamma T}+\frac{\left(s+\gamma D_{T} q^{2}\right)}{c_{s} q} \frac{\dot{\psi}}{c_{s} q}\right]\left\{\frac{1}{\left[1+(\Delta-G)^{2}\right.}\right\} } \\
& \times\left[\frac{1+i(\Delta-G)}{2\left(s+\Gamma q^{2}-i c_{s} q\right)}+\frac{1-i(\Delta-G)}{2\left(s+\Gamma q^{2}+i c_{s} q\right)}\right. \\
& \left.-\frac{1}{\left(s+D_{T} q^{2}\right.}\right],
\end{aligned}
$$

where $\Delta \equiv D_{\top} \mathrm{q} / \mathrm{c}_{\mathrm{s}}$ and $\mathrm{G} \equiv \Gamma \mathrm{q} / \mathrm{c}_{\mathrm{s}}$ are the fraction of the thermal and the acoustic perturbations with a wave vector $q$ that diffuse during the period of the wave, respectively. Typically, $(\Delta, \mathbf{G}) \ll 1$. The three terms in the expansion correspond to three acoustic structures generated optoacoustically. The first two describe isentropic density structures that decay at the classical acoustic damping rate and translate at the sound speed. In this paper, each of these propagating structures is called a phonon for notational convenience, although both structures are actually collections of many coherently excited phonons. The third term describes a stationary isobaric density grating that decays by heat conduction. In this paper, this structure is called a thermon by analogy with the phonons. An upcoming paper will address the concept and the appropriateness of the term thermon. The formation and the shape of the phonons and the thermon depend on the driving terms, $T$ and $\dot{\psi}$. The physics of these results is discussed after expressions are derived for the optoacoustic forcing terms, $T$ and $\dot{\psi}$.

\section{Modeling of Optoacoustic Forcing}

Two optoacoustic effects have been observed in LITA, el ectrostriction and thermalization (see Refs. 1 and 8 for a physical description of both). Electrostriction, in which short driver pulses are used, forces the momentum equation by impulsively accelerating gas molecules toward or away from regions of high opticalfield intensity, depending on the sign of the real part of the susceptibility. Thermalization, which adds heat to the gas, forces the energy equation. This analysis does not consider other optoacoustic effects, although LITA with photophoresis and photolysis presents interesting possibilities for the measurement of physical properties.

The forcing terms for the governing equations are fluid response operators acting on the driver-laser fields. Evaluation of the operators involves modeling of the physical optoacoustic interaction. In this analysis the fluid response operators act on narrowband Gaussian-profile driver beams. The assumed laser beam field is separable into the product of a spatial term and a temporal term. Furthermore, only effects associated with the interference between 
the driver beams are pursued, as only these contribute directly to the LITA signal. Noninterference terms, however, can indirectly modify the signal. For example, bulk heating of a gas by absorption raises the temperature and thus raises the sound speed.

Denote the electric fields of the two driver lasers, $E_{d 1}(x, y, z, t)$ and $E_{d 2}(x, y, z, t)$, so that

$$
\mathrm{E}_{\mathrm{d} 1}=\mathrm{E}_{1}+\mathrm{E}_{1}^{*}, \quad \mathrm{E}_{\mathrm{d} 2}=\mathrm{E}_{2}+\mathrm{E}_{2}^{*},
$$

where* denotes complex conjugation, and

$$
\begin{aligned}
& E_{1}(x, y, z, t)=\frac{\mathscr{E}(t)}{2}\left(\frac{2}{\pi w^{2}}\right)^{1 / 2} \\
& \times \exp \left[-\frac{(y \cos \theta-x \sin \theta)^{2}+z^{2}}{w^{2}}\right] \\
& \times \exp \mathrm{i}\left[\omega_{\mathrm{d}} \mathrm{t}-\mathrm{k}_{\mathrm{d}}(\mathrm{x} \cos \theta+\mathrm{y} \sin \theta]\right. \text {, } \\
& E_{2}(x, y, z, t)=\frac{\mathscr{E}(t)}{2}\left(\frac{2}{\pi w^{2}}\right)^{1 / 2} \\
& x \exp \left[-\frac{(y \cos \theta+x \sin \theta)^{2}+z^{2}}{w^{2}}\right] \\
& \times \exp i\left[\omega_{d} t-k_{d}(x \cos \theta-y \sin \theta)\right],
\end{aligned}
$$

where $x, y$, and $z$ are coor dinates of a Cartesian frame whose $x$ axis is the bisector of the two beams and whose $y$ axis is normal to the bisector in the plane of intersection. $\quad \mathscr{E}(\mathrm{t})$ is the electric-fiel d envel ope, $\omega_{\mathrm{d}}$ is the driver-laser frequency, $k_{d}$ is the wave-vector magnitude of the driver laser, $\theta$ is the beam-crossing half-angle, and $w$ is the Gaussian half-width. The derivation in Subsection 3.A. requires the driverbeam intensity grating and its spatial Fourier transform. Defining the phase-matched scattering or grating vector $\mathrm{q}_{\phi}=2 \mathrm{k}_{d} \sin \theta$ and the grating dimensions $\mathrm{I} \equiv \mathrm{w} / \sin \theta$ and $\mathrm{h} \equiv \mathrm{w} / \cos \theta$

$$
\mathrm{E}_{1}^{*}(\mathbf{r}, \mathrm{t}) \mathrm{E}_{2}(\mathbf{r}, \mathrm{t})+\mathrm{E}_{2}^{*}(\mathbf{r}, \mathrm{t}) \mathrm{E}_{1}(\mathbf{r}, \mathrm{t})=\mathrm{E}_{\mathrm{d}} \mathrm{I}_{\mathrm{d}}(\mathbf{r}) \mathrm{P}_{\mathrm{d}}(\mathrm{t}),
$$

where $E_{d}$ is the total energy of the driver-laser field, $\mathrm{P}_{\mathrm{d}}(\mathrm{t})=\mathscr{E}^{2}(\mathrm{t}) / 2 \mathrm{E}_{\mathrm{d}}$ is the normalized driver-laser intensity history, and $I_{d}(\mathbf{r})$ is the normal ized grating intensity distribution, we find that

$$
\mathrm{I}_{\mathrm{d}}(\mathbf{r})=\frac{2}{\pi \mathrm{w}^{2}} \exp \left[-2\left(\frac{\mathrm{x}^{2}}{\mathrm{I}^{2}}+\frac{\mathrm{y}^{2}}{\mathrm{~h}^{2}}+\frac{\mathrm{z}^{2}}{\mathrm{w}^{2}}\right)\right] \cos \left(\mathrm{q}_{\phi} \mathrm{y}\right) .
$$

The spatial Fourier transform of $\mathrm{I}_{\mathrm{d}}(\mathbf{r})$ is

$$
\begin{aligned}
I_{d}(\mathbf{q})= & \frac{\sqrt{2 \pi} I h}{4 w} \exp \left(-\frac{I^{2} q_{x}^{2}}{8}-\frac{w^{2} q_{z}^{2}}{8}\right) \\
& \times\left(\exp \left[-\frac{h^{2}\left(q_{y}-q_{\phi}\right)^{2}}{8}\right]+\exp \left[-\frac{h^{2}\left(q_{y}+q_{\phi}\right)^{2}}{8}\right]\right)
\end{aligned}
$$

The interference grating is simply a Gaussian ellipsoid with a sinusoidal modulation in the y direction. Its Fourier transform is a pair of Gaussian lobes centered $q_{y}= \pm q_{\phi}$. The spacing of the lobes is proportional to the number of gratings fringes. The width of the lobes is inversely proportional to the diameter of the driver beams. The optoacoustic effects generate susceptibility gratings that initially have the shape of the field intensity grating.

\section{A. Electrostriction Model}

Electrostriction is the tendency of polarizable molecules to move under the action of a potential field caused by the interaction of an optically induced dipolemoment and the optical field. ${ }^{8}$ The energy of a dipole in a electric field is $u(t)=-\epsilon_{0} \mathbf{E}(t) \cdot \mu_{m}(t)$, where $\mathbf{E}(\mathrm{t})$ is the instantaneous el ectric-fiel d vector and $\boldsymbol{\mu}_{\mathrm{m}}(\mathrm{t})$ is the instantaneous molecular dipole moment. A macroscopic ensemble average of this energy over a random distribution of dipole moments sums to 0 . However, a macroscopic dipole moment, $\boldsymbol{\mu}$, may be induced by a second applied el ectric field (or the same field). If $\boldsymbol{\mu}$ and $\mathbf{E}$ are in phase, then the molecules experience a potential field proportional and opposite to the electric-field amplitude. The gradient of this potential accelerates these molecules toward regions of high electric field. The opposite occurs when $\boldsymbol{\mu}$ and $\mathbf{E}$ are out of phase. In terms of the molecular susceptibility, $\chi_{m}$, the el ectrostrictive energy density, $\mathrm{U}_{\mathrm{e}}$, to second order in field strength induced by an optical field $\mathrm{E} \cos (\omega \mathrm{t})$, is

$$
U_{e}(t)=-\operatorname{Re}\left\{\chi_{m}(\omega)\right) \epsilon_{0}\left(E^{2} / 2\right),
$$

where optical-frequency terms have been ignored. In a multispecies gas, the effective electrostrictive potential is proportional to the gas susceptibility, $\chi=$ $\left(\epsilon / \epsilon_{0}-1\right)$, where $\epsilon$ is the local permittivity of the gas and $\epsilon_{0}$ is the permittivity of empty space.

When Doppler shifts from fluid velocity are considered, the electrostrictive potential field experienced by a gas in a LITA experiment is proportional to the average of the real part of the gas susceptibility at the apparent frequencies of the two beams. Each driver beam induces a dipole moment oscillation on which the el ectric field of the other driver beam acts. Other effects are higher order in the driver fields.

Electrostriction may be expressed as a fluid response to an electrostrictive pressure, $p_{e}=U_{e}(t)$, that directly forces the momentum equation through the term $\psi_{\mathrm{e}}=\nabla^{2} \mathrm{p}_{\mathrm{e}} / \rho$, where the subscript e denotes electrostriction. Hence the electrostriction fluid response operator acting on the driver-beam interference pattern is

$$
\dot{\psi}_{\mathrm{e}}(\mathbf{r}, \mathrm{t})=-\frac{\operatorname{Re}\left\{\chi\left(\omega_{\mathrm{d}}\right)\right\}}{\rho} \nabla^{2}\left[\mathrm{E}_{\mathrm{d}} \mathrm{l}_{\mathrm{d}}(\mathbf{r}) \mathrm{P}_{\mathrm{d}}(\mathrm{t})\right],
$$

where $I_{d}=E_{1} E_{2}+E_{2} E_{1}$, and $\mathbf{r}$ is the position vector. Taking the spatial Fourier transform and the temporal Laplace transform of Eq. (9) provides the forcing 
term for Eqs. (1b) and(4):

$$
\dot{\psi}_{\mathrm{e}}(\mathbf{q}, \mathbf{s})=-\frac{\operatorname{Re}\left[\chi\left(\omega_{d}\right)\right\}}{\rho} q^{2} E_{d} I_{d}(\mathbf{q}) P_{d}(\mathbf{s}) .
$$

\section{B. Thermalization Model}

Thermalization describes the process by which driverlaser energy is absorbed by fluid molecules and converted to thermal energy through inelastic collisions. A formal treatment of thermalization dynamics is complicated. The equations of evolution of excitedstate populations could be considered as governing equations for LITA, as they certainly are for degenerate four-wave mixing and CARS. However, in LITA, it is unnecessary to model the energy transfer and the excited-state populations accurately, provided that the thermal energy addition rate is modeled accurately on the LITA time scales. At atmospheric conditions the dynamics of thermalization cannot be completely ignored because quenching of ordinary el ectronic states typically occurs over $0.1-100 \mathrm{~ns}$, and typical Brillouin periods (LITA time scales) are of the order of 10-100 ns. As collision rates increase, thermalization dynamics decrease in importance until the forcing may be treated as a delta function, with no resolvable temporal structure.

The second-order density-operator perturbation theory indicates that the rate of driver-laser-induced population of excited states is proportional to the negative imaginary part of the susceptibility, i.e., the linear absorption coefficient. 8 The overpopulation of the excited states is assumed to decay to the ground state by superelastic (thermalization) collisions at a rate $\gamma_{\theta}$ and by other processes at a rate $\gamma_{n \theta}$. The rate of thermal energy addition to the fluid is $\partial \mathrm{U}_{\theta} / \partial \mathrm{t}=$ $\gamma_{\theta} U_{e x}$, where the subscript $\theta$ denotes thermalization. The excited-state energy density, $U_{e x}$, obeys the field equation

$$
\begin{aligned}
\frac{\partial \mathrm{U}_{\mathrm{ex}}}{\partial \mathrm{t}}+\gamma_{\theta} \mathrm{U}_{\mathrm{ex}}+\gamma_{n \theta} \mathrm{U}_{\mathrm{ex}}- & \mathrm{D}_{\mathrm{s}} \nabla^{2} \mathrm{U}_{\mathrm{ex}} \\
& \left.=-2 \mathrm{k}_{\mathrm{d}} \operatorname{Im}\left[\chi\left(\omega_{\mathrm{d}}\right)\right]\left[\mathrm{E}_{\mathrm{d}} \mathrm{P}_{\mathrm{d}}(\mathrm{t})\right)_{\mathrm{d}}(\mathbf{r})\right], \\
\mathrm{U}_{\mathrm{ex}}(\mathbf{r}, \mathrm{t}=0) & =0 .
\end{aligned}
$$

$D_{s}$ is the diffusivity of excited-state mol ecules through the gas. The excited-state energy grating induced by narrow-bandwidth driver beams in a moving fluid is proportional to the absorption coefficient at the apparent frequencies of the driver beams. Physically, this means that the excited-state grating is being population through the interaction of each driver beam with the macroscopic dipole moment induced by the other driver beam.

Taking the spatial Fourier transform and temporal Laplace transform of Eq. (11) and substituting the expression for $\mathrm{U}_{\theta}$ provides

$$
\mathrm{U}_{\theta}(\mathbf{q}, \mathrm{s})=\frac{-2 \gamma_{\theta} \mathrm{k}_{\mathrm{d}} \operatorname{Im}\left(\chi\left(\omega_{\mathrm{d}}\right)\right) \mathrm{E}_{\mathrm{d}} \mathrm{l}_{\mathrm{d}}(\mathbf{q}) \mathrm{P}(\mathrm{s})}{\mathrm{s}\left(\mathrm{s}+\gamma_{\mathrm{n} \theta}+\gamma_{\theta}+\mathrm{D}_{\mathrm{s}} \mathrm{q}^{2}\right)}
$$

The rate of temperature rise is $\dot{\mathrm{T}}(\mathbf{q}, \mathrm{s})=\mathrm{sU}_{\theta}(\mathbf{q}, \mathrm{s}) / \rho \mathrm{c}_{\mathrm{v}}$; hence the fluid response operator for thermalization acting on the driver-beam interference pattern is

$$
\dot{T}(\mathbf{q}, \mathbf{s})=\frac{-2 \gamma_{\theta} k_{d} \operatorname{Im}\left(\chi\left(\omega_{d}\right)\right) E_{d} I_{d}(\mathbf{q}) P(s)}{\rho c_{v}\left(s+\gamma_{n \theta}+\gamma_{\theta}+D_{s} q^{2}\right)} .
$$

More realistic thermalization models are simple to device and incorporate by the generalization of

$$
\dot{\mathrm{T}}(\mathbf{q}, \mathbf{s})=\frac{-2 \mathrm{k}_{\mathrm{d}} \operatorname{Im}\left(\chi\left(\omega_{\mathrm{d}}\right)\right) \mathrm{E}_{\mathrm{d}} \mathrm{l}_{\mathrm{d}}(\mathbf{q}) \mathrm{P}_{\mathrm{d}}(\mathrm{s}) \Theta(\mathrm{q}, \mathrm{s})}{\rho \mathrm{c}_{\mathrm{v}}},
$$

where $\Theta(q, s)$ models the energy transfer. However, the single-rate thermalization model elucidates the physics, matches actual behavior with reasonable accuracy, and serves as a basis for improved models, ${ }^{1}$ e.g., $\Theta(q, s) \simeq \Sigma_{i} a_{i} \gamma_{\theta, i}\left(s+\gamma_{n \theta, i}+\gamma_{\theta, i}+D_{s, i} q^{2}\right)^{-1}$.

This physical modeling of optoacoustic forcing is valid only if local collisional equilibration occurs on a time scale that is short compared with time scales of interest. This caveat is consistent with the requirement that the LITA grating Knudsen number be small, which is needed for the hydrodynamic equations (1a)-(1c) to be valid and the quasi-continuum concepts of pressure, density, sound speed, and transport properties to be valid on the LITA length scale.

Combining Eqs. (4), (10), and (13a), we obtain an expression for the Fourier and the Laplace transforms of the optoacoustic density fields in terms of driver-laser and fluid parameters;

$$
\begin{aligned}
\frac{\rho_{l}(\mathbf{q}, \mathrm{s})}{\rho}= & \mathrm{I}_{\mathrm{d}}(\mathbf{q}) \mathrm{P}_{\mathrm{d}}(\mathrm{s}) \mathrm{E}_{\mathrm{d}}\left[\frac{-2 \mathrm{k}_{\mathrm{d}} \gamma_{\theta} \operatorname{Im}\left\{\chi\left(\omega_{\mathrm{d}}\right)\right\}}{\rho \mathrm{c}_{\mathrm{p}} \mathrm{T}}\right. \\
& \times \frac{1}{\left(\mathrm{~s}+\gamma_{\mathrm{n}} \theta+\gamma \theta+\mathrm{D}_{\mathrm{s}} \mathrm{q}^{2}\right)}-\frac{\operatorname{Re}\left\{\chi\left(\omega_{\mathrm{d}}\right)\right\}}{\rho \mathrm{c}_{\mathrm{s}}^{2}} \\
& \left.\times\left(\mathrm{s}+\gamma \mathrm{D}_{\mathrm{T}} \mathrm{q}^{2}\right)\right]\left(\frac{1}{\left[1+(\Delta-\mathrm{G})^{2}\right]}\right) \\
& \times\left[\frac{1+\mathrm{i}(\Delta-\mathrm{G})}{2\left(\mathrm{~s}+\Gamma \mathrm{q}^{2}-\mathrm{i} \mathrm{c}_{\mathrm{s}} \mathrm{q}\right)}+\frac{1+\mathrm{i}(\Delta-\mathrm{G})}{2\left(\mathrm{~s}+\Gamma \mathrm{q}^{2}+\mathrm{i} \mathrm{c}_{\mathrm{s}} \mathrm{q}\right)}\right. \\
& \left.-\frac{1}{\left(\mathrm{~s}+\mathrm{D}_{\mathrm{T}} \mathrm{q}^{2}\right)}\right]
\end{aligned}
$$

Laplace inversion is aided by another partial fractions expansion. Define

$$
\begin{aligned}
& u_{\theta}=-\frac{2 k_{d} \operatorname{lm}\left\{\chi\left(\omega_{d}\right)\right\} E_{d}}{w^{2} \rho c_{p} T} \frac{\gamma_{\theta}}{\gamma_{n \theta}+\gamma_{\theta}+D_{s} q^{2}}, \\
& u_{e}=-\frac{q \operatorname{Re}\left(\chi\left(\omega_{d}\right)\right\} E_{d}}{w^{2} \rho c_{s}^{2}} .
\end{aligned}
$$

$U_{\theta}$ and $U_{e}$ may be considered as the approximate modulation depths of the thermalization and the electrostriction gratings, respectively. Let the sym- 
bol denote Laplace convolution:

$$
f(t) g(t) \equiv \int_{0}^{\infty} f(\tau) g(t-\tau) d \tau
$$

The density field time behavior is

$$
\frac{\rho_{1}(\mathbf{q}, t)}{\rho}=-w^{2} I_{d}(\mathbf{q}) P_{d}(t)\left[H_{\theta}(q, t) u_{\theta}+H_{e}(q, t) u_{e}\right] .
$$

Hence the temporal behavior of the optoacoustic gratings is a convolution over the laser time history of $\mathrm{H}_{\theta}$ and $\mathrm{H}_{\mathrm{e}}$, which may be regarded as Green's functions in time for the fluid response to thermalization and el ectrostriction, respectively, which are given by

$$
\begin{aligned}
H_{\theta}(q, t) \equiv & H_{\theta P}(q) \Phi_{P}(q, t)+H_{\theta P}^{*}(q) \Phi_{P}^{*}(q, t) \\
& +H_{\theta T}(q) \Phi_{T}(q, t)+H_{\theta D}(q) \Phi_{D}(q, t), \\
H_{e}(q, t) \equiv & H_{\theta P}(q) \Phi_{P}(q, t)+H_{\theta^{P}}^{*}(q) \Phi_{P}^{*}(q, t) \\
& +H_{e T}(q) \Phi_{T}(q, t),
\end{aligned}
$$

where

$$
\begin{aligned}
\Phi_{P}(q, t) & \equiv \exp \left(-\Gamma q^{2} t+i c_{s} q t\right), \Phi_{T}(q, t) \equiv \exp \left(-D_{T} q^{2} t\right), \\
\Phi_{D}(q, t) & \equiv \exp \left[-\left(\gamma_{\theta}+\gamma_{n \theta}\right) t-D_{s} q^{2} t\right], \\
H_{\theta P} & \equiv \frac{[1+i(\Delta-G)(1-G \Pi-i \Pi)}{\left.2\left[1+(\Delta-G)^{2}\right](1-G \Pi)^{2}+\Pi^{2}\right]}, \\
H_{\theta T} & \equiv \frac{-1}{\left[1+(\Delta-G)^{2}\right](1-\Delta \Pi)}, \\
H_{\theta D} & \equiv \frac{\Pi^{2}}{\left[(1-G \Pi)^{2}+\Pi^{2}\right](1-\Delta \Pi)}, \\
H_{e P} & \equiv i \frac{[1-i(\gamma \Delta-G)[1+i(\Delta-G)]}{2\left[1+(\Delta-G)^{2}\right]}, \\
H_{e T} & \equiv \frac{(\gamma-1) \Delta}{\left[1+(\Delta-G)^{2}\right]}, \\
\Pi & \equiv c_{s} q /\left(\gamma_{\theta}+\gamma_{n \theta}+D_{s} q^{2}\right) .
\end{aligned}
$$

The parameter $\Pi$ is the ratio of the frequency of sound waves with wave vector $\mathrm{q}$ to the excited-state energy decay rate. $\Pi$ should be small for efficient phonon formation. In the limit of fast thermalization and negligible damping over a wave period, the parameters $\mathrm{H}_{\theta_{\mathrm{p}}} \rightarrow 1 / 2, \mathrm{H}_{\theta_{\mathrm{T}}} \rightarrow-1, \mathrm{H}_{\theta_{\mathrm{D}}} \rightarrow 1, \mathrm{H}_{\mathrm{eP}} \rightarrow$ $\mathrm{i} / 2$, and $\mathrm{H}_{\mathrm{eT}} \rightarrow 0$. At atmospheric conditions, $\Delta$ and $\Gamma \sim 0.4 / \lambda_{g}$, where $\lambda_{g}$ is the grating wavelength expressed in micrometers; hence, for typical grating spacings of 5-50 $\mu \mathrm{m}$, these parameters are negligible. Under the same conditions, $\Pi \sim 2 / \lambda_{g}-50 / \lambda_{g}$, depending on the quenching rates of the excited state, and therefore may not be negligible.
The Fourier transform of the driver grating, $I_{d}(\mathbf{q})$, is strongly peaked at $\mathbf{q}=q_{\phi} \hat{e}_{y}$, where $\hat{e}_{y}$ is the unit vector in the $q_{y}$ direction. Assume therefore that the coefficients of the elements $\mathrm{H}_{\theta, P, T, D}$ and $\mathrm{H}_{\mathrm{e} P, \mathrm{~T}\}}$ are equal to their values at $q=q_{\phi}$. Also, to second order in $q / q_{\phi}$, approximate $q \simeq q_{y}+\left(q_{x}^{2}+q_{z}^{2}\right) / 2 q_{p}$. The inverse Fourier transforms of the products $I_{d}(\mathbf{q}) \Phi_{[P, T, D]}$ $(\mathrm{q}, \mathrm{t}) \equiv \Phi_{[\mathrm{P}, \mathrm{T}, \mathrm{D}\}}^{\mathrm{d})}$ are, without further approximation,

$$
\begin{aligned}
& \Phi_{\mathrm{P}}^{\mathrm{d}}(\mathbf{r})=\frac{2 \mathrm{lh}}{\pi \mathrm{W} \Lambda_{\mathrm{P}} \Omega_{\mathrm{P}} \mathrm{H}_{\mathrm{P}}} \\
& \times \exp \left[-2 \frac{x^{2}}{\Lambda_{P}^{2}}-2 \frac{z^{2}}{\Omega_{P}^{2}}-2 \frac{\left(y+c_{s} t\right)^{2}}{H_{P}^{2}} \Gamma q_{\phi}^{2} t \eta_{P}^{2}\right] \\
& \times \cos \left[\left(y+c_{s} t\right) q_{\phi} \eta_{P}^{2}\right], \\
& \Phi_{\mathrm{P} *}^{\mathrm{d}}(\mathbf{r})=\frac{2 \mathrm{lh}}{\pi \mathrm{W} \Lambda_{\mathrm{P}}^{*} \Omega_{\mathrm{p}}^{*} \mathrm{H}_{\mathrm{P}}} \\
& \times \exp \left[-2 \frac{\mathrm{x}^{2}}{\Lambda_{\mathrm{P}}^{* 2}}-2 \frac{\mathrm{z}^{2}}{\Omega_{\mathrm{P}}^{* 2}}-2 \frac{\left(\mathrm{y}-\mathrm{c}_{\mathrm{s}} \mathrm{t}\right)^{2}}{\mathrm{H}_{\mathrm{P}}^{2}} \Gamma \mathrm{q}_{\phi}^{2} \operatorname{t\eta }_{\mathrm{P}}^{2}\right] \\
& \times \cos \left[\left(y-c_{s} t\right) q_{\phi} \eta_{P}^{2}\right] \text {, } \\
& \Phi_{\mathrm{T}}^{(\mathrm{d})}(\mathbf{r})=\frac{2 \mathrm{lh}}{\pi \mathrm{W} \Lambda_{\mathrm{T}} \Omega_{\mathrm{T}} \mathrm{H}_{\mathrm{T}}} \\
& \times \exp \left(-2 \frac{\mathrm{x}^{2}}{\Lambda_{\mathrm{T}}^{2}}-2 \frac{\mathrm{y}^{2}}{\mathrm{H}_{\mathrm{T}}^{2}}-2 \frac{\mathrm{z}^{2}}{\Omega_{\mathrm{T}}^{2}}-\mathrm{D}_{\mathrm{T}} \mathrm{q}_{\Phi}^{2} \mathrm{t} \eta_{\mathrm{T}}^{2}\right) \\
& \times \cos \left(y q_{\phi} \eta_{T}^{2}\right) \text {, } \\
& \Phi_{\mathrm{D}}^{(\mathrm{d})}(\mathbf{r})=\frac{2 \mathrm{l} h}{\pi \mathrm{w} \Lambda_{\mathrm{D}} \Omega_{\mathrm{D}} \mathrm{H}_{\mathrm{D}}} \\
& \times \exp \left[-2 \frac{x^{2}}{\Lambda_{D}^{2}}-2 \frac{y^{2}}{H_{D}^{2}}-2 \frac{z^{2}}{\Omega_{D}^{2}}-D_{s} q_{\phi}^{2} \operatorname{t\eta }_{D}^{2}\right. \\
& \left.-\left(\gamma_{\theta}+\gamma_{n \theta}\right) t\right] \cos \left(y q_{\phi} \eta_{D}^{2}\right) \text {, }
\end{aligned}
$$

(A18d)

where

$$
\begin{aligned}
\Lambda_{\mathrm{P}}^{2} & \equiv \mathrm{I}^{2}+8 \Gamma \mathrm{t}-4 i \mathrm{c}_{\mathrm{S}} \mathrm{t} / \mathrm{q}_{\phi}, \quad \Lambda_{\mathrm{T}}^{2} \equiv \mathrm{I}^{2}+8 \mathrm{D}_{\mathrm{T}} \mathrm{t}, \\
\Lambda_{\mathrm{D}}^{2} & \equiv \mathrm{I}^{2}+8 \mathrm{D}_{\mathrm{s}} \mathrm{t}, \quad \mathrm{H}_{\mathrm{P}}^{2} \equiv \mathrm{h}^{2}+8 \Gamma \mathrm{t}, \quad \mathrm{H}_{\mathrm{T}}^{2} \equiv \mathrm{h}^{2}+8 \mathrm{D}_{\mathrm{T}} \mathrm{t}, \\
\mathrm{H}_{\mathrm{D}}^{2} & \equiv \mathrm{h}^{2}+8 \mathrm{D}_{\mathrm{s}} \mathrm{t}, \quad \Omega_{\mathrm{P}}^{2} \equiv \mathrm{w}^{2}+8 \Gamma \mathrm{t}-4 \mathrm{i} \mathrm{c}_{\mathrm{S}} \mathrm{t} / \mathrm{q}_{\phi}, \\
\Omega_{\mathrm{T}}^{2} & \equiv \mathrm{w}^{2}+8 \mathrm{D}_{\mathrm{T}} \mathrm{t}, \quad \Omega_{\mathrm{D}}^{2} \equiv \mathrm{w}^{2}+8 \mathrm{D}_{\mathrm{s}} \mathrm{t}, \\
\eta_{\mathrm{P}} & \equiv \mathrm{h} / \mathrm{H}_{\mathrm{P}}, \quad \eta_{\mathrm{T}} \equiv \mathrm{h} / \mathrm{H}_{\mathrm{T}}, \quad \eta_{\mathrm{D}} \equiv \mathrm{h} / \mathrm{H}_{\mathrm{D}} .
\end{aligned}
$$

This relatively complicated nomenclature results from all the dimensions of each grating changing at different rates. Note that, except for gratings with very few fringes, the LITA signals will decay before these new length scales depart significantly from their values at $\mathrm{t}=0$, with one exception: $\Omega_{\mathrm{P}}$, for which the imaginary part increases relative to the real part at a rate $c_{s} / w$. Thus, normally, the spreading of the 
grating envel ope by diffusion may be ignored but the wave motion cannot.

\section{Discussion of Grating Formation and Evolution}

The results of the derivation of expressions for the creation and the evolution of acoustic grating by electrostriction and thermalization bear discussion. The creation and the evolution of the gratings may be understood simply by first considering the situation in which the optoacoustic driving occurs instantly. Consider the properties of the fluid across the grating at cross section A-A in Fig. 1 at time $t=0$, immediately after driving occurs by the use of a deltafunction laser pulse. If thermalization occurs instantly, the temperature perturbation follows the el ectric-field intensity grating. However, at timet $=$ 0 there has been no bulk displacement of the gas; thus no density grating exists. The gas is in a nonequilibrium state because, at equilibrium, the density is inversely proportional to temperature. The response of the gas may be explained in terms of the thermon and the phonons. The flat density profile at $t=0$ is the superposition of the equilibrium density field borne by the thermon and two opposing density fields borne by two conjugate phonons. At $t=0$ the field of the phonons cancels the field of the thermon. At $t>0$, the motion of the phonons modulates the density field as the phonons alternately interfere constructively and destructively with the thermon. The frequency of the density field modulation, the Brillouin frequency $\omega_{B}$, is $\omega_{B}=c_{s} q_{\phi}$. As the phonons propagate, they decay (rigorously, the number of

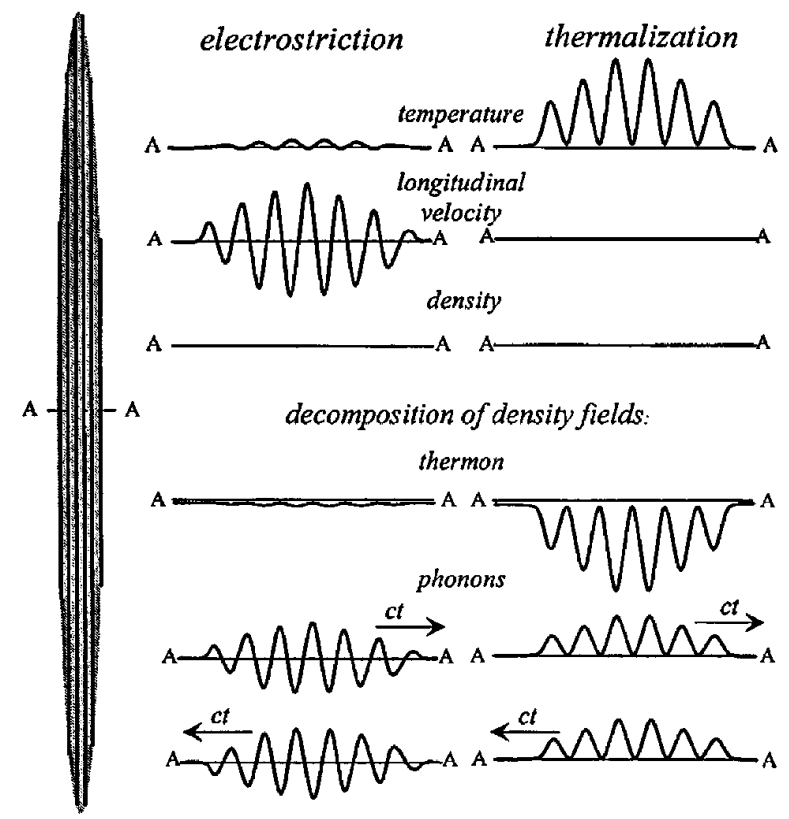

Fig. 1. Optoacoustic generation of phonons and thermons. The curves are field profiles al ong the cross section A-A of the grating, shown at left. Laser driving and thermalization are assumed to be instantaneous. At time $t=0$ the density fields of the phonons and the thermon sum to 0 . Afterward, motion of the phonons and damping modulate the density grating amplitude. coherent phonons comprising the acoustic wave packets decays) by diffusion at the acoustic damping rate. The thermon decays by thermal diffusion. The assumption of linear hydrodynamic behavior eliminates a variety of effects that could affect signals in real experiments. For example, the thermon will perturb the local sound speed, causing coupling between the phonons and the thermons. Limiting the modulation depth of the grating suitably, e.g., $<1 \%$, mitigates nonlinear effects.

Instantaneous electrostriction impulsively accelerates the target molecules toward or away from regions of high electric field. By (assumed instantaneous) Iocal equilibration of molecular velocities, the target molecules transfer momentum to the bulk gas, creating a velocity grating. The velocity grating induces density gratings. Again the density fields sum to 0 at $t=0$. In electrostriction, the phonons typically dominatethe thermon. Furthermore, these phonons are $\pm 90^{\circ}$ out of phase with the phonons generated by thermalization. The temporal modulation of the density grating occurs primarily at twice the Brillouin frequency.

When driving time scales are comparable with the hydrodynamics scales, the response is more complicated. First, the temporal behavior of the acoustic gratings is convolved with the driver-laser time history, which effectively smears the phonons and steepens the thermons (over the freely diffusing profile). Second, thermalization introduces another temporal convolution. In the current model, the convolution kernel is a simple exponential decay. For more realistic models the convolution is over a more complicated kernel. Convolution over the thermalization alsosmears the phonons. For efficient phonon formation, driver-laser durations and thermalization times must be short compared with the inverse of the Brillouin frequency. If thermalization rates are sufficiently fast, the acoustic structures become independent of the details of the thermalization behavior, simplyfing the analysis of LITA signals.

At atmospheric pressure, collision rates are $\sim 10^{9}$ $10^{10} / \mathrm{s}$. If the ambient gas quenches the excited state of the target species reasonably well, the smallest expected thermalization times are $\sim 1-20 \mathrm{~ns}$. The largest practical grating spacings are $\sim 30 \mu \mathrm{m}$, yielding a period of motion across a grating wavelength of $\sim 100$ ns in room-temperature air. Thus at STP, finitethermalization rates may be important contributors to the LITA signal, as indicated above. The absence of independent means of measuring the thermalization behavior is a significant detriment to the verification of the analysis. Narrow-band timeresolved LITA tests are being performed in a highpressure optical bomb in which thermalization-rate effects can be addressed.

\section{Modeling of Acousto-Optic Scattering}

Nonuniformities of a gas scatter light. Rayleigh and Brillouin scattering arise from statistical isobaric and 
isentropic fluctuations of the gas density, respectivel $\mathrm{y}^{4}$ These scattering processes are weak because the fluctuations are incoherent. The acousto-optic effects that create the LITA signal beam are the same as those of theselinear scattering phenomena (except for scattering caused by species concentration gratings). However, LITA scattering may be many orders of magnitudestronger than Rayleigh or Brillouin scattering because the fluctuations are coherent. ${ }^{4,6}$ This coherent enhancement of the LITA signal over classical linear scattering techniques does not change the spectrum of the scattered light but only the intensity. The spectrum of the LITA signal scattered from a broadband laser off a density grating is the same as the Rayleigh-Brillouin spectrum of the gas in the sample volume (except with extremely broadband source lasers, for which phase matching becomes a concern). Hence the LITA reflectivity spectrum is far less complicated than those of most other nonlinear optical techniques. The simple reflectivity spectrum motivates the use of LITA for multiplex spectral measurements.

The next step in the analysis is the treatment of acousto-optics by the linearized equations of light scattering. In the far field, the optical el ectric field $\mathrm{E}_{\mathrm{s}}$ scattered by a small disturbance in the (isotropic) susceptibility $\chi_{1}(\mathbf{r}, \mathrm{t} ; \omega)$ from a narrow-band source beam characterized by $E_{0}(r, t) \cos \left(\omega_{0} t\right)$, with a slowly varying envel ope, is ${ }^{9}$

$$
\mathrm{E}_{\mathrm{s}}(\mathbf{R}, \mathrm{t} ; \mathbf{q})=-\frac{\mathrm{k}_{\mathrm{s}}^{2}}{4 \pi \mathrm{R}} \cos \left(\mathbf{k}_{\mathrm{s}} \cdot \mathbf{R}-\omega_{\mathrm{o}} \mathrm{t}\right) \mu_{\mathrm{l}}(\mathbf{q}, \mathrm{t}),
$$

where $\mathbf{q}$ is the change in wave vector from the source to the scattered beam, $k_{s}$ is the wave vector of the scattered beam $\left(\left|k_{s}\right| \simeq\left|k_{o}\right|\right), \mathbf{R}$ is the position vector relative to the scatterer, and the function $\mu_{1}(\mathbf{q}, t)$ is the spatial F ourier transform of the overlap, $\mu_{1}(\mathbf{r}, t)$, of the susceptibility grating and the source laser field, i.e.,

$$
\mu_{1}(\mathbf{r}, \mathrm{t})=\chi_{1}\left(\mathbf{r}, \mathrm{t} ; \omega_{0}\right) \mathrm{E}_{\mathrm{o}}(\mathbf{r}, \mathrm{t}) .
$$

The change in gas susceptibility caused by the optoacoustic effects comes from a combination of the change in density, temperature, and species concentration. The dependence of the susceptibility on density changes is usually the strongest acousto-optic effect for gases and is the only effect treated in this analysis. The gas susceptibility is roughly linear with density for small density perturbations, $\chi_{1} \simeq \chi \rho_{1} / \rho$. Thus the scattered field $\mathrm{E}_{\mathrm{s}}$ is known in terms of the density perturbation field, the source laser profile, and the linear susceptibility at the source laser frequency.

For this simple analysis, consider a narrow-band, Gaussian source beam with half-width $\sigma$, negligible divergence, and center frequency $\omega_{0}$, coplanar with the driver lasers and incident upon the sample volume at the phase-matched angle, $\phi$. Furthermore, assume that the source beam is perfectly centered on the sample volume. Denote, $\sigma_{x} \equiv \sigma / \sin \phi$ and $\sigma_{y} \equiv$ $\sigma / \cos \phi$. The el ectric fiel $d$ of the source beam is then $\mathrm{E}_{0}(\mathbf{r}, \mathrm{t})=\mathrm{P}_{0}(\mathrm{t}) \mathrm{I}_{0}(\mathbf{r}) \cos \left(\omega_{0} \mathrm{t}\right)$, where

$$
\mathrm{I}_{\mathrm{o}}=\left(\frac{2}{\pi \sigma^{2}}\right)^{1 / 2} \exp \left[-\left(\frac{\mathrm{y}}{\sigma_{\mathrm{y}}}-\frac{\mathrm{x}}{\sigma_{\mathrm{x}}}\right)^{2}-\frac{\mathrm{z}}{\sigma}\right] ;
$$

$\mathrm{I}_{\mathrm{o}}$ is normalized so that the instantaneous power of the source laser is $\mathrm{P}_{0}^{2}(\mathrm{t})$. Define $\Phi_{\mathrm{P}, T, \mathrm{O}}^{\mathrm{d}, \mathrm{o}}(\mathbf{r}, \mathrm{t}) \equiv$ $I_{d}(\mathbf{r}) \Phi_{P, T, D}^{d d}(\mathbf{r}, t)$, the field of the overlap of the source beam and the subscripted acoustic structures. The Fourier transforms of $\Phi_{\mathrm{P}, \mathrm{O}, \mathrm{D}}^{\mathrm{d}, \mathrm{d}}$ dictate the LITA scattering lobe shapes and time dependence. The component of the scattering vector $q_{x}$ is assumed to be 0 for all scattered light. The Fourier transform in $\mathrm{x}$ becomes the integral of $\Phi_{P, T, D}^{d, o}(\mathbf{r}, \mathbf{t})$ over $\mathbf{x}$. The overlap length of the source beam and the grating in the $x$ direction is typically $\sim 10-30$ times that in the $y$ and the $z$ directions. Hence the finite size effects in the $x$ direction may be safely ignored. One accomplishes this by letting $\sigma_{\mathrm{x}} \rightarrow \infty$. Performing the integration and the transforms over $y$ and $z$ in this limit and ignoring the contribution from the lobe at $q_{y}=-q_{\phi}$ one obtains

$$
\begin{aligned}
\Phi_{\mathrm{P}}^{(\mathrm{d}, \mathrm{o}}(\mathbf{q}) & =\mathrm{N}_{\mathrm{P}} \Psi_{\mathrm{P}}(\mathbf{q}) \Sigma_{\mathrm{P}}(\mathrm{t}), \quad \Phi_{\mathrm{T}}^{(\mathrm{d}, \mathrm{o})}=\mathrm{N}_{\mathrm{T}} \Psi_{\mathrm{T}}(\mathbf{q}) \Sigma_{\mathrm{T}}(\mathrm{t}), \\
\Phi_{\mathrm{D}}^{(\mathrm{d}, \mathrm{o})} & =\mathrm{N}_{\mathrm{D}} \Psi_{\mathrm{D}}(\mathbf{q}) \Sigma_{\mathrm{D}}(\mathrm{t}),
\end{aligned}
$$

where

$$
\begin{aligned}
& \mathrm{N}_{\mathrm{P}} \equiv \frac{\left(\pi \xi_{\mathrm{P}} \zeta_{\mathrm{P}}\right)^{1 / 2} \mathrm{Ih}}{\mathrm{W} \sigma \mathrm{H}_{\mathrm{P}} \Omega_{\mathrm{P}}}, \quad \mathrm{N}_{\mathrm{T}} \equiv \frac{\left(\pi \xi_{\mathrm{T}} \zeta_{\mathrm{T}}\right)^{1 / 2} \mathrm{Ih}}{\mathrm{W} \sigma \mathrm{H}_{\mathrm{T}} \Omega_{\mathrm{T}}}, \\
& \mathrm{N}_{\mathrm{D}} \equiv \frac{\left(\pi \xi_{\mathrm{D}} \zeta_{\mathrm{D}}\right)^{1 / 2} \mathrm{Ih}}{\mathrm{W} \sigma \mathrm{H}_{\mathrm{D}} \Omega_{\mathrm{D}}} \\
& \Psi_{\mathrm{P}} \equiv\left(\frac{\xi_{\mathrm{P}} \zeta_{\mathrm{P}}}{4 \pi}\right)^{1 / 2} \exp \left(-\frac{\xi_{\mathrm{P}}^{2} \mathrm{q}_{\mathrm{y}}^{\prime 2}}{8}-\frac{\zeta_{\mathrm{P}}^{2} \mathrm{q}_{\mathrm{z}}^{2}}{8}+\frac{\xi_{\mathrm{P}}^{2}}{\mathrm{H}_{\mathrm{P}}^{2}} \mathrm{i} \mathrm{c}_{\mathrm{s}} \mathrm{q}_{\mathrm{y}}^{\prime} \mathrm{t}\right), \\
& \Psi_{\mathrm{T}} \equiv\left(\frac{\xi_{\mathrm{T}} \zeta_{\mathrm{T}}}{8 \pi}\right)^{1 / 2} \exp \left(-\frac{\xi_{\mathrm{T}}^{2} \mathrm{q}_{\mathrm{y}}^{\prime 2}}{8}-\frac{\zeta_{\mathrm{T}}^{2} \mathrm{q}_{\mathrm{z}}^{2}}{8}\right), \\
& \Psi_{\mathrm{D}} \equiv\left(\frac{\xi_{\mathrm{D}} \zeta_{\mathrm{D}}}{8 \pi}\right)^{1 / 2} \exp \left(-\frac{\xi_{\mathrm{D}}^{2} \mathrm{q}_{\mathrm{y}}^{\prime 2}}{8}-\frac{\zeta_{\mathrm{D}}^{2} q_{\mathrm{z}}^{2}}{8}\right) \text {, } \\
& \Sigma_{P} \equiv \exp \left(-\frac{2 c_{S}^{2} t^{2}}{H_{P}^{2}+2 \sigma_{y}^{2}}-\Gamma q_{\phi}^{2} t \eta_{P}^{2}+i q_{\phi} c_{S} t_{P}^{2}\right), \\
& \Sigma_{\mathrm{T}} \equiv \exp \left(-\mathrm{D}_{\mathrm{T}} \mathrm{q}_{\phi}^{2} \mathrm{t}_{\mathrm{T}}^{2}\right) \text {, } \\
& \Sigma_{D} \equiv \exp \left[-D_{s} q_{\phi}^{2} t \eta_{D}^{2}-\left(\gamma_{\theta}+\gamma_{n \theta}\right) t\right],
\end{aligned}
$$

where $q_{y, P, T, D]}^{\prime} \equiv q_{y}-q_{\phi} \eta_{P, T, D]}^{2}$, and

$$
\begin{aligned}
& \xi_{\{P, T, D]} \equiv\left(\frac{1}{H_{[P, T, D]}^{2}}+\frac{1}{2 \sigma_{y}^{2}}\right)^{-1 / 2}, \\
& \zeta_{\{P, T, D]} \equiv\left(\frac{1}{\Omega_{[P, T, D\}}^{2}}+\frac{1}{2 \sigma^{2}}\right)^{-1 / 2}
\end{aligned}
$$


are overlap length scales in the $y$ and the $z$ directions.

Substituting these expressions into Eq. (19a) provides the scattered field of the LITA signal:

$$
\begin{aligned}
& \frac{E_{s}(\mathbf{q}, R, t)}{P_{0}(t)}=-\frac{k_{s}^{2} w^{2}}{4 \pi R} \chi\left(\omega_{0}\right) \exp i\left(k_{s} \cdot \mathbf{R}-\omega_{0} t\right) P_{d}(t) \\
& {\left[2 \operatorname{Re}\left\{\Phi_{\mathrm{P}}^{\mathrm{d}, \mathrm{o}}(\mathbf{q}, \mathrm{t})\left(\boldsymbol{u}_{\theta} \mathrm{H}_{\theta \mathrm{P}}+\boldsymbol{u}_{\mathrm{e}} \mathrm{H}_{\ominus \mathrm{P}}\right)\right\}\right.} \\
& +\Phi_{\mathrm{T}}^{\mathrm{d}, \mathrm{o}}(\mathbf{q}, \mathrm{t})\left(\mathcal{U}_{\theta} \mathrm{H}_{\theta \mathrm{T}}+u_{\mathrm{e}} \mathrm{H}_{\mathrm{eT}}\right) \\
& \left.+\Phi_{\mathrm{D}}^{\mathrm{d}, \mathrm{o}}(\mathbf{q}, \mathrm{t}) \mathrm{U}_{\theta} \mathrm{H}_{\theta \mathrm{D}}\right] \text {. }
\end{aligned}
$$

\section{Integration to Obtain the LITA Signal}

The LITA signal obtained by the use of heterodyne detection is equal to the integral of the field [Eq. (22)] over the detection angle (a range of $\mathrm{q}_{y}^{\prime}$ and $\mathrm{q}_{z}$ centered on 0 ). If this range is infinitesimal, the detected signal is given by Eq. (22). If the detection angle is larger than the lobe size (large-detector limit), these integrals may be approximated by infinite integrals, giving the result for $\mathscr{L}_{\text {het, }}$ the heterodyne LITA signal:

$$
\begin{aligned}
& \frac{\mathscr{L}_{\text {het }}}{\left.\mathrm{P}_{\mathrm{o}} \mathrm{t}\right)}=\sqrt{\pi} \frac{\mathrm{lh}}{\mathrm{W \sigma}} \chi\left(\omega_{0}\right) \frac{\mathrm{k}_{\mathrm{o}}^{2} \mathrm{w}^{2}}{4 \pi} \\
& \times \exp \left(-i \omega_{0} t\right) P_{d}(t) / \operatorname{Re} \frac{\left(\left(\xi_{P} \zeta_{P}\right)^{1 / 2}\right.}{H_{P} \Omega_{P}} \\
& \left.\times \exp \left(-\frac{2 c_{S}^{2} t^{2}}{H_{P}^{2}}-\Gamma q_{\phi}^{2} t \eta_{P}^{2}+i q_{\phi} c_{s} t \eta_{P}^{2}\right)\right) \\
& \left.\times\left(u_{\theta} \mathrm{H}_{\theta \mathrm{P}}+u_{\mathrm{e}} \mathrm{H}_{\Theta P}\right)\right\}+\frac{\left(\xi_{\mathrm{T}} \zeta_{\mathrm{T}}\right)^{1 / 2}}{\mathrm{H}_{\mathrm{T}} \Omega_{\mathrm{T}}} \\
& \times \exp \left(-\mathrm{D}_{\mathrm{T}} \mathrm{q}_{\phi}^{2} \mathrm{t}_{\mathrm{T}}^{2}\right)\left(u_{\theta} \mathrm{H}_{\theta \mathrm{T}}+u_{\mathrm{e}} \mathrm{H}_{\mathrm{eT}}\right)+\frac{\left(\xi_{\mathrm{D}} \zeta_{\mathrm{D}}\right)^{1 / 2}}{\mathrm{H}_{\mathrm{D}} \Omega_{\mathrm{D}}} \\
& \left.\times \exp \left[-D_{s} q_{\phi}^{2} \operatorname{t\eta } \eta_{D}^{2}-\left(\gamma_{\theta}+\gamma_{n \theta}\right) t\right] u_{\theta} H_{\theta D}\right),
\end{aligned}
$$

where $k_{s}$ is assumed to be equal to $k_{0}$. Note the interesting result that the signal from the phonons drops like $\exp \left[-\left(\mathrm{c}_{\mathrm{S}} \mathrm{t} / \mathrm{H}_{\mathrm{P}}\right)^{2}\right]$ as the phonons propagate. The phonons behave as if they scatter light onto the large, phase-sensitive detector only whilethey overlap. A physical interpretation is that light scattered by the phonons is out of phase when they do not overlap, canceling the signal observed on the detector. When the detector size is small compared with the scattering lobe size, the light scattered by the phonons adds up in phase over the entire source beam, regardless of whether the phonons overlap. The physical picture that provides the most insight into the phonon signal is different in both limits. In the large-detector limit, the notion that the phonons scatter light only when they overlap, thus creating an oscillatory standing-wave pattern, leads to the proper conclusions about the envelope of the signal. In the small- detector limit, the envelope of the phonon signal appears to be Doppler beats between light scattered by independent moving gratings. Because many of the potential applications of LITA involve interpreting the time-resolved signal, it is important to be aware of these detector effects.

In homodyne experiments, the detected signal is the integral of the square of the modulus of the scattered field over the detection angle. Define the amplitudes of the phonon, thermon, and the finitedriving-time terms:

$$
\begin{aligned}
& A_{P} \equiv u_{\theta} H_{\theta P}+u_{e} H_{e P}, \quad A_{T} \equiv u_{\theta} H_{\theta T}+u_{e} H_{e T}, \\
& A_{D} \equiv u_{\theta} H_{\theta D} .
\end{aligned}
$$

If the detector is larger than the signal beam size, these integrals may be approximated by infinite integrals over $q_{y}^{\prime}$ and $q_{z}$. The element of area of the detector is dydz $=\left[\mathrm{R}^{2} /\left(\mathrm{k}_{0} \cos \phi\right)^{2}\right] \mathrm{dq}_{\mathrm{y}} \mathrm{dq}_{\mathrm{z}}$. The result of this integration appears in Appendix A. One may simplify the complete result in most instances by ignoring the change in length scales that is due to diffusion, but wave motion cannot be ignored. For notational convenience, regard the driver laser as a del ta function in time, thereby eliminating the convolutions. The complete expression, which is given in Eq. (A1), simplifies to

$$
\begin{aligned}
& \frac{\mathscr{L}_{\text {hom }}}{\mathrm{P}_{\mathrm{o}}^{2}(\mathrm{t})}=\frac{\pi}{4} \frac{\mathrm{I}^{2}}{\lambda_{\mathrm{o}}^{2}}\left|\chi\left(\omega_{\mathrm{o}}\right)\right|^{2} \frac{\xi \zeta}{\sigma^{2} \cos ^{2} \phi \cos ^{2} \theta}\left(\operatorname{Re} 2 \mathrm{~A}_{\mathrm{P}}^{2}\left(\frac{\mathrm{w}^{2} \zeta_{\mathrm{P}}}{\Omega_{\mathrm{P}}^{2} \zeta}\right)\right. \\
& \times \exp \left(-\frac{4 c_{s}^{2} t^{2}}{h^{2}+2 \sigma_{y}^{2}}-2 \Gamma q_{\phi}^{2} t+2 i q_{\phi} c_{s} t\right) \\
& \times \exp \left(\frac{-4 \xi^{2} c_{s}^{2} t^{2}}{h^{4}}\right)+4 A_{P} A_{T}\left[\left(\frac{2 \zeta_{P}^{2}}{\zeta_{P}^{2}+\zeta^{2}}\right)\right]^{1 / 2} \frac{w}{\Omega_{P}} \\
& \times \exp \left[-\frac{2 c_{s}^{2} t^{2}}{h^{2}+2 \sigma_{y}^{2}}-\left(D_{T}+\Gamma\right) q_{\theta}^{2} t+i q_{\phi} c_{s} t\right] \\
& \times \exp \left(\frac{-\xi^{2} c_{S}^{2} t^{2}}{h^{4}}\right)+4 A_{P} A_{D}\left[\left(\frac{2 \zeta_{P}^{2}}{\zeta_{P}^{2}+\zeta^{2}}\right)\right]^{1 / 2} \frac{w}{\Omega_{P}} \\
& \times \exp \left[-\frac{2 c_{s}^{2} t^{2}}{h^{2}+2 \sigma_{y}^{2}}-\left(D_{s}+\Gamma\right) q_{\phi}^{2} t+i q_{\phi} c_{s} t\right. \\
& \left.\left.-\left(\gamma_{\theta}+\gamma_{n \theta}\right) t\right] \exp \left(\frac{-\xi^{2} c_{s}^{2} t^{2}}{h^{4}}\right)\right\}+2 A_{P} A \stackrel{*}{*}\left(\frac{w^{2} \zeta_{P}}{\Omega_{p}^{2} \zeta}\right) \\
& \times \exp \left(-\frac{4 c_{s}^{2} t^{2}}{h^{2}+2 \sigma_{y}^{2}}-2 \Gamma q_{\phi}^{2} t\right)+2 A_{T} A_{D} \\
& \times \exp \left[-\left(D_{\mathrm{T}}+D_{s}\right) q_{\phi}^{2} t-\left(\gamma_{\theta}+\gamma_{n \theta}\right) t\right] \\
& +A_{T}^{2} \exp \left(-2 D_{T} q_{\phi}^{2} t\right) \\
& \left.+A_{D}^{2} \exp \left[-2 D_{s} q_{\phi}^{2} t-2\left(\gamma_{\theta}+\gamma_{n \theta}\right) t\right]\right),
\end{aligned}
$$


where $\lambda_{0}$ is the source of laser wavel ength and

$$
\xi \equiv\left(\frac{1}{h^{2}}+\frac{1}{2 \sigma_{y}^{2}}\right)^{-1 / 2}, \quad \zeta \equiv\left(\frac{1}{w^{2}}+\frac{1}{2 \sigma^{2}}\right)^{-1 / 2} .
$$

Because beam-crossing angles are usually small, the distinction between $\xi$ and $\zeta$ can usually beignored.

When the source beam is large compared with all other LITA length scales and the beam-crossing angle is small, Eq. (25a) may be simplified further:

$$
\begin{aligned}
\frac{\mathscr{L}_{\text {hom }}}{\mathrm{P}_{0}^{2}(t)}= & \frac{\pi}{4}\left(\frac{I}{\lambda_{0}}\right)^{2}\left|\chi\left(\omega_{0}\right)\right|^{2}\left(\frac{w}{\sigma}\right)^{2} \\
& \times\left(\operatorname { R e } \left\{2 A_{P}^{2} \exp \left(-2 \Gamma q_{\phi}^{2} t+2 i q_{\phi} c_{s} t-\frac{4 c_{s}^{2} t^{2}}{w^{2}}\right)\right.\right. \\
& +4 A_{P} A_{T} \exp \left[-\left(D_{T}+\Gamma\right) q_{\phi}^{2} t+i q_{\phi} c_{s} t-\frac{c_{s}^{2} t^{2}}{w^{2}}\right] \\
& +4 A_{P} A_{D} \exp \left[-\left(D_{s}+\Gamma\right) q_{\phi}^{2} t+i q_{\phi} c_{s} t\right. \\
& \left.\left.-\left(\gamma_{\theta}+\gamma_{n \theta}\right) t-\frac{\xi^{2} c_{s}^{2} t^{2}}{h^{4}}\right]\right)+2 A_{P} A_{P}^{*} \\
& \times \exp \left(-2 \Gamma q_{\phi}^{2} t\right)+2 A_{T} A_{D} \\
& \times \exp \left[-\left(D_{T}+D_{s}\right) q_{\phi}^{2} t-\left(\gamma_{\theta}+\gamma_{n \theta}\right) t\right] \\
& +A_{T}^{2} \exp \left(-2 D_{T} q_{\phi}^{2} t\right)+A_{D}^{2} \\
& \times \exp \left[-2 D_{s} q_{\phi}^{2} t-2\left(\gamma_{\theta}+\gamma_{n \theta} t\right]\right) .
\end{aligned}
$$

Equations (23), (25a), (26), and (A1) are equalities that are valid in their respective limits and thus may predict absolute signal levels from assumed grating modulation depths $\left(u_{\theta}\right.$ and $u_{\mathrm{e}}$. They provide estimates of the absolute signal strength, given a known susceptibility spectrum. These estimates are only approximate because the modeling to obtain $u_{\theta}$ and $u_{\mathrm{e}}$ in terms of the gas susceptibility assumes a monochromatic driver laser. A more realistic model of the laser-gas interaction is relatively simple to insert into the analysis.

The time history of the homodyne-detected signal depends on the detection angle. The effect is different from that in heterodyne detection. Because the detection is not phase sensitive, the signals scattered by the phonons after they travel away from each other do not cancel. However, the Doppler beating between the signals scattered by the phonons is a heterodyne effect. Thus, when the detection angle is large, the amplitude of the Doppler beats falls as the phonons travel away from each other. The homodyne signal drops as the phonons move away from the source beam. As happens in a heterodyne detection of LITA signals, when the detection angle is small, the Doppler beats fade by motion of the phonons away from the source beam.

The behavior of Doppler beats in the LITA signal, a classical interference, is entirely analogous of those of quantum interferences. For example, quantummechanical scattering interferences arise from in principle indeterminacy in the path of the scattered particle. In LITA, the interference arises because of uncertainty in the origin of the scattered light while the phonons overlap. As the phonons move away from each other, the uncertainty and the Doppler beats fade. A small detector has inadequate spatial resolution in principle to determine the origin of the scattered light and therefore sees Doppler beats even after the phonons have moved away from each other.

The LITA signal reflectivity has a $\left|\chi\left(\omega_{0}\right)\right|^{2}$ spectrum that resembles linear scattering that is considerably simpler than those of other nonlinear multiplex techniques, such as CARS $^{6}$ and DFWM. ${ }^{10}$ Broadband source beams with intensities low enough to prevent saturation may be used to measure these linear spectra in a single laser shot.

\section{Comparison of Theoretical and Experimental LITA Signals}

Single-shot, narrow-band, time-resolved LITA signals have been measured with a N d:YAG-pumped (590-nm) dye laser for the driver beams, a cw dye laser for the source beam, and laboratory air as the test gas. The details of these experiments are given in Ref. 1. LITA signals from both thermalization and electrostriction were measured as a function of driverlaser wavelength, beam size, and grating wavelength.

An interesting dependence of the LITA signal on driver-laser frequency was observed in tests performed on laboratory air. The behavior was caused by spectral structure in the gas susceptibility from $\mathrm{NO}_{2}$ that was present in the air in quantities of less than 50 parts in $10^{9}$ according to the Southern California Air-Quality Management District, which monitors atmospheric pollutants. Because electrostriction and thermalization depend on different components of the susceptibility, the rel ative contribution to the signals from each effect changes as the driver-laser frequency is tuned across spectral lines or structure. Figure 2 illustrates this effect for a hypothetical transition. When a monochromatic driver-laser frequency is far from the transition (a), the signal arises predominantly from nonresonant electrostriction. The signal oscillates at twice the Brillouin frequency because of Doppler beating between light scattered by the counterpropagating phonons. Near the transition frequency, the real part of the susceptibility attains a maximum (b). The electrostrictive contribution to the signal increases. A staircase pattern emerges in which every other peak is enhanced. The increase in these peaks arises from a component of the signal at the Brillouin frequency, which heralds the formation of a thermon. At the peak of the absorption (c), the signal arises almost entirely from thermalization. The electrostrictive peaks return totheir nonresonant level. At a slightly higher frequency $(d)$, the real part of the susceptibil- 

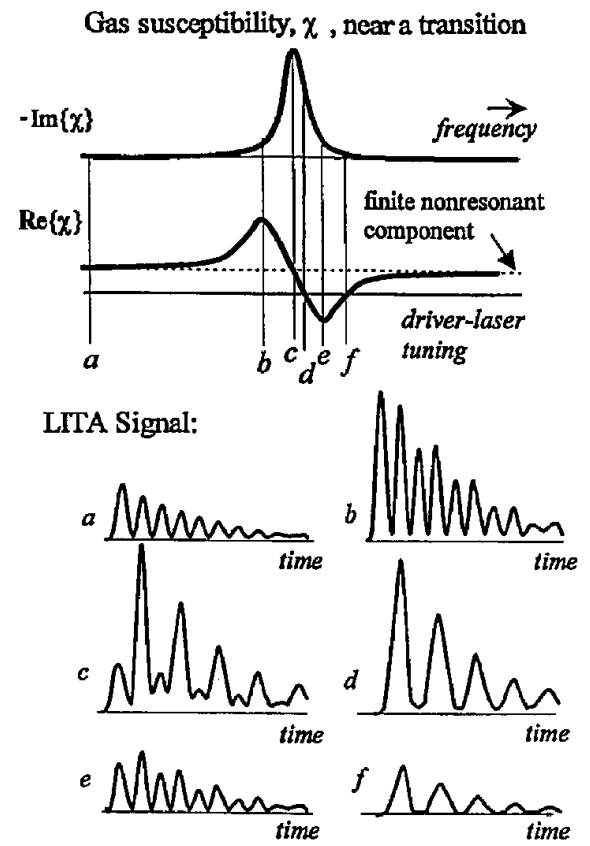

Fig. 2. LITA signal versus driver-laser tuning. Electrostriction, proportional to $\operatorname{Re}\left\{\left(\omega_{d}\right)\right\}$, produces a signal modulated at twice the Brillouin frequency. Thermalization, proportional to $-\operatorname{Im}\left\{\chi\left(\omega_{d}\right)\right)$, produces a signal modulated at the Brillouin frequency.

ity is nulled. Thesignal arises entirely from thermalization, as it does at $f$. At e, there is a slight return of electrostriction peaks. N ormomonochromaticity of the driver laser complicates the dependence of electrostriction and thermalization on gas susceptibility. The effect is discussed in Ref. 11.

A dependence of the LITA signal on driver-laser frequency, like that shown in Fig. 2, was observed in LITA signals from the laboratory air. It is unlikely, however, that an isolated line of $\mathrm{NO}_{2}$ (as in Fig. 2) was probed because of the high density of transitions in the spectral region that was probed. Four illustrative data sets showing the effect of driver-laser tuning and their theoretical fits appear in Figs. 3-6. The accompanying theoretical fits use published values for the diffusivities and calculated sound speeds and assume that the detector is large compared with the scattering lobe. The fitting parameters include the thermalization rate and the relative strengths of the electrostriction and the thermalization signals. Beam widths and crossing angles inferred from the experimental data are far more precise than independent measurements and are used consistently for the theoretical fits. The signals are convolved over an approximate laser pulse shape ( $\sim 5-n s$ FWHM). The signals in Figs. 3-6 are normalized to the peak of the signal in Fig. 3.

Figure 3 illustrates a signal obtained when the driver beam frequency matched that of an absorption peak. The signals in Figs. 3-6 are ensemble averaged over 256 shots to reduce shot noise. This averaging smears the signal somewhat as a result of shot-to-shot variations, drift, jitter, and laser noise. There was no independent way to quantify these

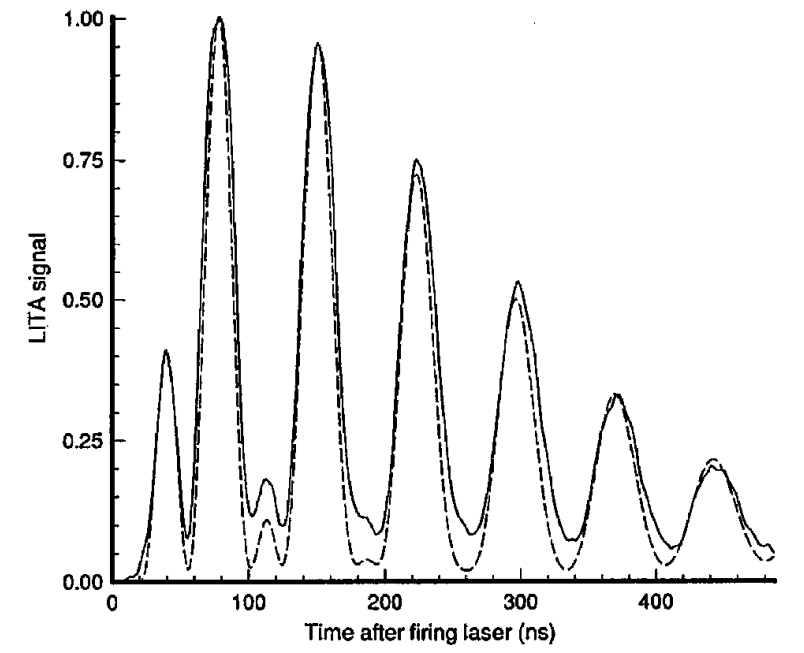

Fig. 3. Experimental LITA signal and theoretical fit by the use of Eqs. (A1) with published calculated properties of atmospheric air, $\mathrm{w}=162 \mu \mathrm{m}, \sigma=173 \mu \mathrm{m}, \mathrm{q}_{\phi}=2 \pi / 25.3 \mu \mathrm{m}$. The driver-laser frequency is tuned to an absorption peak of $\mathrm{NO}_{2}$, present in concentrations of $<50$ parts in $10^{9}$.

effects, so there was no attempt to convolve the signal over an additional noise kernel to improve the fit. Slight beam misalignments may also have affected the signal envelope.

Figures 4 and 5 show signals in which el ectrostriction and thermalization contributions are comparable. Figure 6 shows a signal in which the dominant optoacoustic effect is nonresonant electrostriction. The theoretical curves clearly capture the physics of theinteraction. Discrepancies between the data and the fit are attributed to non-Gaussian beam profiles, nonideal beam overlap, multiple-rate thermalization behavior, laser noise, experimental noise, drift, and jitter. Future work in narrow-band LITA includes further verification of the accuracy of the theoretical

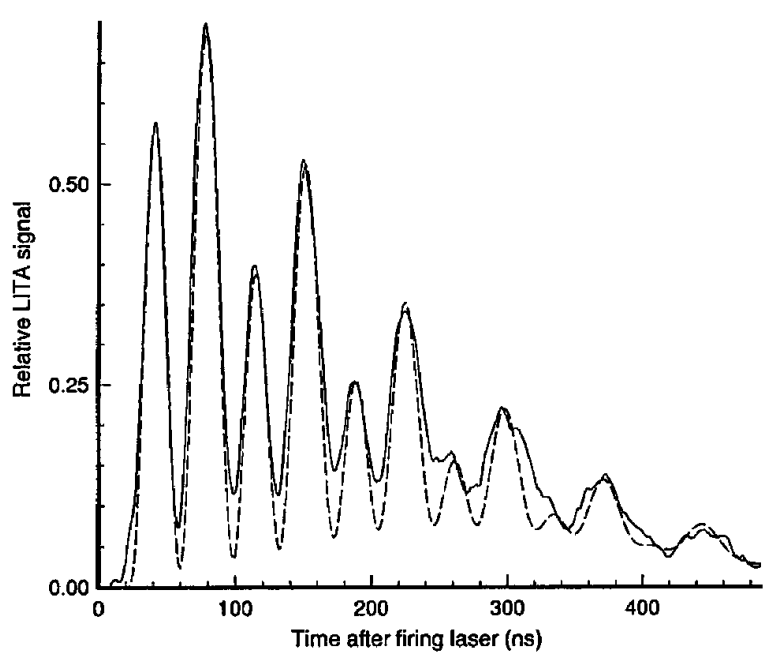

Fig. 4. Experimental LITA signal and theoretical fit. Thedriverlaser frequency is $36 \mathrm{GHz}$ higher than that for the data in Fig. 3. All other parameters are the same as in Fig. 3. The only fitting parameters are $\varkappa_{\theta}, u_{\mathrm{e}}$, and $\gamma_{\theta}$. The signal is normalized with respect to the signal in Fig. 3. 


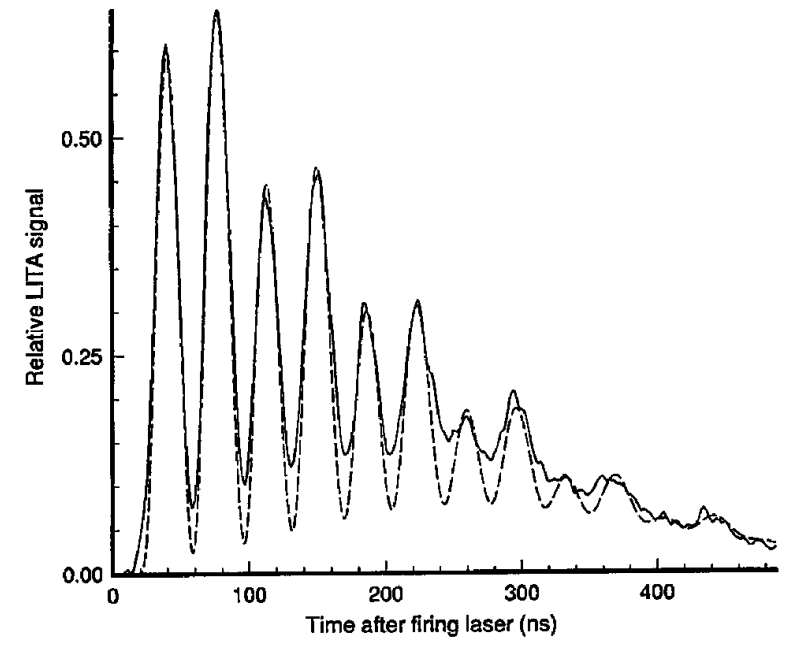

Fig. 5. Experimental LITA signal and theoretical fit. Thedriverlaser frequency is $72 \mathrm{GHz}$ higher than that for the data in Fig. 3. Otherwise, the description is the same as for Fig. 4.

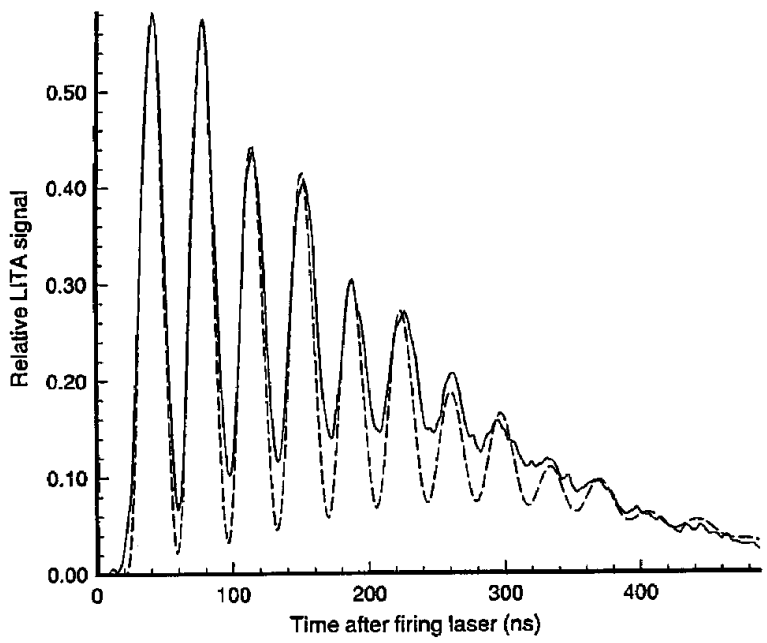

Fig. 6. Experimental LITA signal and theoretical fit. Thedriverlaser frequency is $120 \mathrm{GHz}$ higher than that for the data in Fig. 3. Otherwise, the description is the same as for Fig. 4.

model, incorporation of higher-order mode structure, and modeling and elimination of the nonidealities responsible for differences between theory and experiment.

\section{Conclusions}

An analytical expression for the time history and the absolute magnitude of the LITA signal has been derived for finite-diameter Gaussian driver and source beams. This model closely matches experimental data and may be used both to predict and to interpret LITA signals. The temporal history of the detected signal depends on the size of the detection angle. This result has implications for researchers who hope to measure diffusivities by using time-resolved LITA. The LITA reflectivity has a $\left|\chi^{(1)}\right|^{2}$ spectrum like that of Rayleigh-Brillouin scattering. Multiplex LITA, which exploits this feature, is a promising technique for single-shot species concentration and temperature measurement.

\section{Appendix A.}

The complete expression for the homodyne-detected LITA signal, including all the diffusion terms and the convolutions over the driver-beam pulse shapes, is

$$
\begin{aligned}
& \frac{\mathscr{L}_{\text {hom }}}{\mathrm{P}_{\mathrm{o}}^{2}(\mathrm{t})}=\frac{\mathrm{k}_{\mathrm{o}}^{2} \mathrm{w}^{4}}{16 \pi^{2} \cos ^{2} \phi}\left|\chi\left(\omega_{\mathrm{o}}\right)\right|^{2} \int_{0}^{\infty} \int_{0}^{\infty} \mathrm{P}_{\mathrm{d}}\left(\mathrm{t}-\tau_{1}\right) \mathrm{P}_{\mathrm{d}}\left(\mathrm{t}-\tau_{2}\right) \\
& \times\left[\operatorname { R e } \left\{2 A_{P}^{2} N_{P}\left(\tau_{1}\right) N_{P}\left(\tau_{2}\right)\right.\right. \\
& \times\left(\frac{4 \zeta_{\mathrm{P}}\left(\tau_{1}\right) \xi_{\mathrm{P}}\left(\tau_{1}\right) \zeta_{\mathrm{P}}\left(\tau_{2}\right) \xi_{\mathrm{P}}\left(\tau_{2}\right)}{\left[\xi_{\mathrm{P}}^{2}\left(\tau_{1}\right)+\xi_{\mathrm{P}}^{2}\left(\tau_{2}\right)\right]\left[\zeta_{\mathrm{P}}^{2}\left(\tau_{1}\right)+\zeta_{\mathrm{P}}^{2}\left(\tau_{2}\right)\right]}\right)^{1 / 2} \\
& \times \exp \left(-2 c_{S}^{2}\left[\frac{\tau_{1}^{2} \xi_{P}^{2}\left(\tau_{1}\right)}{H_{P}^{4}\left(\tau_{1}\right)}+\frac{\tau_{2}^{2} \xi_{P}^{2}\left(\tau_{2}\right)}{H_{P}^{4}\left(\tau_{2}\right)}\right) \Sigma_{P}\left(\tau_{1}\right) \Sigma_{P}\left(\tau_{2}\right)\right. \\
& +4 \mathrm{~A}_{\mathrm{P}} \mathrm{A}_{\mathrm{T}} \mathrm{N}_{\mathrm{P}}\left(\tau_{1}\right) \mathrm{N}_{\mathrm{T}}\left(\tau_{2}\right) \\
& \times\left(\frac{4 \zeta_{\mathrm{P}}\left(\tau_{1}\right) \xi_{\mathrm{P}}\left(\tau_{1}\right) \xi_{T}\left(\tau_{2}\right) \xi_{T}\left(\tau_{2}\right)}{\left[\xi_{\mathrm{P}}^{2}\left(\tau_{1}\right)+\xi_{T}^{2}\left(\tau_{2}\right)\right]\left[\zeta_{\mathrm{P}}^{2}\left(\tau_{1}\right)+\zeta_{T}^{2}\left(\tau_{2}\right)\right]}\right)^{1 / 2} \\
& \times \exp \left(\frac{-2 \xi_{\mathrm{P}}^{4}\left(\tau_{1}\right){c_{\mathrm{S}}^{2}}^{2} \tau_{1}^{2}}{\mathrm{H}_{\mathrm{P}}^{4}\left(\tau_{1}\right)\left[\xi_{\mathrm{P}}^{2}\left(\tau_{1}\right)+\xi_{\mathrm{T}}^{2}\left(\tau_{2}\right)\right]}\right) \Sigma_{\mathrm{P}}\left(\tau_{1}\right) \Sigma_{\mathrm{T}}\left(\tau_{2}\right) \\
& +4 A_{P} A_{D} N_{P}\left(\tau_{1}\right) N_{D}\left(\tau_{2}\right) \\
& \times\left(\frac{4 \zeta_{\mathrm{P}}\left(\tau_{1}\right) \xi_{\mathrm{P}}\left(\tau_{1}\right) \zeta_{\mathrm{D}}\left(\tau_{2}\right) \xi_{\mathrm{D}}\left(\tau_{2}\right)}{\left[\xi_{\mathrm{P}}^{2}\left(\tau_{1}\right)+\xi_{D}^{2}\left(\tau_{2}\right)\right]\left[\zeta_{\mathrm{P}}^{2}\left(\tau_{1}\right)+\zeta_{D}^{2}\left(\tau_{2}\right)\right]}\right)^{1 / 2} \\
& \left.\times \exp \left(\frac{-2 \xi_{P}^{4}\left(\tau_{1}\right) c_{S}^{2} \tau_{1}^{2}}{H_{P}^{4}\left(\tau_{1}\right)\left[\xi_{P}^{2}\left(\tau_{1}\right)+\xi_{D}^{2}\left(\tau_{2}\right)\right.}\right) \Sigma_{P}\left(\tau_{1}\right) \Sigma_{D}\left(\tau_{2}\right)\right\} \\
& +2 A_{P} A_{P}^{*} N_{P}\left(\tau_{1}\right) N_{P}^{*}\left(\tau_{2}\right) \\
& \times\left(\frac{4 \zeta_{P}\left(\tau_{1}\right) \xi_{P}\left(\tau_{1}\right) \zeta_{P}\left(\tau_{2}\right) \xi_{P}\left(\tau_{2}\right)}{\left[\xi_{P}^{2}\left(\tau_{1}\right)+\xi_{P}^{2}\left(\tau_{2}\right)\right]\left[\zeta_{P}^{2}\left(\tau_{1}\right)+\zeta_{P}^{2}\left(\tau_{2}\right)\right]}\right)^{1 / 2} \\
& \times \Sigma_{\mathrm{P}}\left(\tau_{1}\right) \Sigma_{\mathrm{P}}^{*}\left(\tau_{2}\right)+2 \mathrm{~A}_{\mathrm{T}} \mathrm{A}_{\mathrm{D}} \mathrm{N}_{\mathrm{T}}\left(\tau_{1}\right) \mathrm{N}_{\mathrm{D}}\left(\tau_{2}\right) \\
& \times\left(\frac{4 \zeta_{T}\left(\tau_{1}\right) \xi_{T}\left(\tau_{1}\right) \zeta_{D}\left(\tau_{2}\right) \xi_{D}\left(\tau_{2}\right)}{\left[\xi_{T}^{2}\left(\tau_{1}\right)+\xi_{D}^{2}\left(\tau_{2}\right)\right]\left[\zeta_{T}^{2}\left(\tau_{1}\right)+\zeta_{D}^{2}\left(\tau_{2}\right)\right]}\right)^{1 / 2} \\
& \times \Sigma_{\mathrm{T}}\left(\tau_{1}\right) \Sigma_{\mathrm{D}}\left(\tau_{2}\right)+\mathrm{A}_{\mathrm{T}}^{2} \mathrm{~N}_{\mathrm{T}}\left(\tau_{1}\right) \mathrm{N}_{\mathrm{T}}\left(\tau_{2}\right) \\
& \times\left(\frac{4 \zeta_{T}\left(\tau_{1}\right) \xi_{T}\left(\tau_{1}\right) \zeta_{T}\left(\tau_{2}\right) \xi_{T}\left(\tau_{2}\right)}{\left[\xi_{T}^{2}\left(\tau_{1}\right)+\xi_{T}^{2}\left(\tau_{2}\right)\right]\left[\zeta_{T}^{2}\left(\tau_{1}\right)+\zeta_{T}^{2}\left(\tau_{2}\right)\right]}\right)^{1 / 2} \\
& \times \Sigma_{T}\left(\tau_{1}\right) \Sigma_{T}\left(\tau_{2}\right)+A_{D}^{2} N_{D}\left(\tau_{1}\right) N_{D}\left(\tau_{2}\right) \\
& \times\left(\frac{4 \zeta_{D}\left(\tau_{1}\right) \xi_{D}\left(\tau_{1}\right) \zeta_{D}\left(\tau_{2}\right) \xi_{D}\left(\tau_{2}\right)}{\left[\xi_{D}^{2}\left(\tau_{1}\right)+\xi_{D}^{2}\left(\tau_{2}\right)\right]\left[\zeta_{D}^{2}\left(\tau_{1}\right)+\zeta_{D}^{2}\left(\tau_{2}\right)\right]}\right)^{1 / 2} \\
& \times \Sigma_{\mathrm{D}}\left(\tau_{1}\right) \Sigma_{\mathrm{D}}\left(\tau_{2}\right) \mathrm{d} \tau_{1} \mathrm{~d} \tau_{2}
\end{aligned}
$$

This expression applies when $\mathrm{I}^{2} \gg \mathrm{w}^{2}$, i.e., small values of the beam-crossing angle, $\theta$, because finite- 
beam-size effects in the $x$ direction were ignored for brevity. This expression appears to model experimental signals fairly well, even if the driver beams depart significantly from their Gaussian ideal. Accurate source-beam alignment, however, is important, particularly in experiments in which focused beams are used.

The authors gratefully acknowledge the support of the U niversity Research I nitiative in aerothermochemistry sponsored by the U. S. Air Force Office of Scientific Research. E. Cummings is also indebted to the U. S. Office of Naval Research for its support of his graduate education through its fellowship program.

\section{References}

1. E. B. Cummings, "Laser-induced thermal acoustics: simple accurate gas measurements," Opt. Lett. 19, 1361-1363 (1994).

2. D. E. Govoni, J. A. Booze, A. Sinha, and F. F. Crim, "The non-resonant signal in laser-induced grating spectroscopy," Chem. Phys. Lett. 216, 525-529 (1993).

3. S. Williams, L. A. Rahn, P. H. Paul, and J. W. Forsman,
"Laser-induced thermal grating effects in flames," Opt. Lett. 19, 1361-1363 (1994).

4. H. J . Eichler, P. Günter, and D. W. Pohl, Laser-Induced Dynamic Gratings (Springer-Verlag, New York, 1986), pp. 84-91.

5. A. C. Eckbreth, Laser Diagnostics for Combustion Species and Temperature (Abacus, Cambridge, Mass., 1988), pp. 272-277.

6. E. B. Cummings, "Techniques of single-shot thermometry by degenerate four-wave mixing," GALCIT Rep. number FM 92-2 (California Institute of Technology, Pasadena, Calif., 1992).

7. B. J . Berne and R. Pecora, Dynamic Light Scattering (Wiley, New York, 1976), pp. 233-240.

8. R. W. Boyd, Nonlinear Optics (Academic, San Diego, Calif., 1992), pp. 126-143, 327-331.

9. L. D. Landau and E. M. Liftshitz, Electrodynamics of Continuous Media (Addison-Wesley, Cambridge, Mass., 1960), 377383.

10. D. R. Meacher, P. G. R. Smith, and P. Ewart, "Frequency spectrum of the signal wave in resonant four-wave mixing induced by broad-bandwidth lasers," Phys. Rev. A 46, 27182725 (1992).

11. E. B. Cummings, "Laser induced thermal acoustics," M. S. thesis (California Institute of Technology, Pasadena, Calif., 1995), pp. 89-92. 